\title{
THE CYCLIC CONNECTIVITY OF HOMOGENEOUS ARCWISE CONNECTED CONTINUA ${ }^{1}$
}

\author{
BY \\ DAVID P. BELLAMY ${ }^{2}$ AND LEWIS LUM ${ }^{3}$
}

\begin{abstract}
A continuum is cyclicly connected provided each pair of its points lie together on some simple closed curve. In 1927, G. T. Whyburn proved that a locally connected plane continuum is cyclicly connected if and only if it contains no separating points. This theorem was fundamental in his original treatment of cyclic element theory. Since then numerous authors have obtained extensions of Whyburn's theorem. In this paper we characterize cyclic connectedness in the class of all Hausdorff continua.

THEOREM. The Hausdorff continuum $X$ is cyclicly connected if and only if for each point $x \in X, x$ lies in the relative interior of some arc in $X$ and $X-\{x\}$ is arcwise connected.

We then prove that arcwise connected homogeneous metric continua are cyclicly connected.
\end{abstract}

Introduction. In his Ph.D. dissertation [14] R. L. Wilson proved that no uniquely arcwise connected Hausdorff continuum supports the structure of a topological group. Since he used mainly algebraic techniques his proof revealed little about the topological nature of such continua. Indeed, in private conversation with the authors, Wilson asked whether uniquely arcwise connected Hausdorff continua can be homogeneous.

In this paper we characterize cyclicly connected continua; i.e., continua in which each pair of points lie together on a simple closed curve. Arcwise connected homogeneous metric continua are then shown to be cyclicly connected. This yields a negative answer to Wilson's question for metric continua; the nonmetric case remains open.

The authors wish to thank G. R. Gordh, Jr. for many helpful discussions and suggestions during the preparation of this paper.

A Cyclic Connectedness Theorem. The continuum $X$ is cyclicly connected [12] provided each pair of points in $X$ lie together on some simple closed curve in $X$.

Received by the editors February 19, 1979.

1980 Mathematics Subject Classification. Primary 54F20; Secondary 54F65.

${ }^{1}$ A portion of this paper was presented to the American Mathematical Society under the title "Arcwise connected homogeneous continua contain simple closed curves" at the 1980 National Meeting.

${ }^{2}$ The first author was supported during the latter stages of this research by NSF grant number MCS 7908413.

${ }^{3}$ This research was begun while the second author was a visitor at the University of Delaware in 1978-79. He wishes to thank the members of the Mathematical Sciences Department there for their hospitality during that year. 
In [12] G. T. Whyburn proved that a locally connected plane continuum is cyclicly connected if and only if it contains no separating points. Later W. L. Ayres [1] (see also [13] and [2]) proved the same theorem without the planar assumption. The absence of cut points (see [9, p. 188]) was seen, by F. B. Jones [6], to be sufficient for cyclic connectedness in nonseparating plane continua. C. L. Hagopian [4] obtained a sufficient condition for cyclic connectedness in aposyndetic plane continua. The example in Figure 1 is a nonaposyndetic (and hence nonlocally connected) cyclicly connected plane continuum and in Figure 2 we have an aposyndetic noncyclicly connected plane continuum. In this section we characterize cyclic connectedness in the class of all Hausdorff continua.

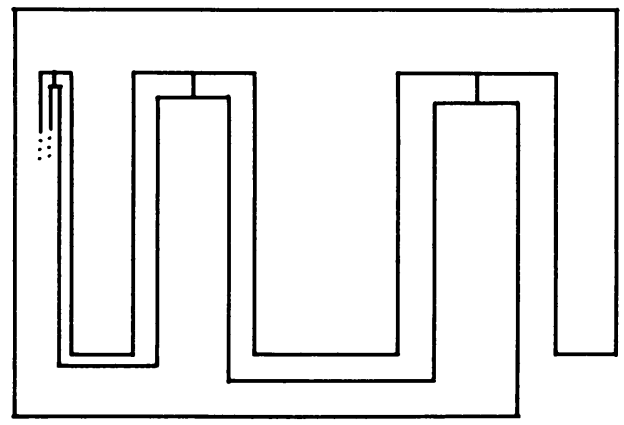

\section{FIGURE 1}

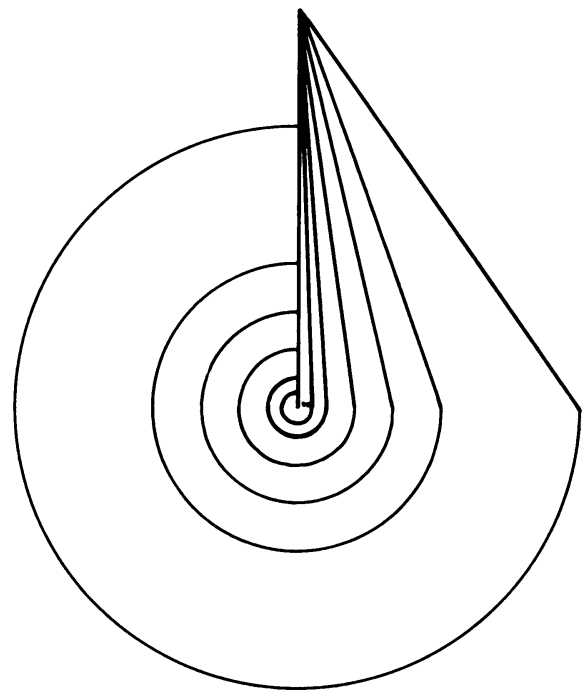

FIGURE 2

In a Hausdorff continuum an arc is a subcontinuum with exactly two nonseparating points and a simple closed curve is a subcontinuum which is the union of two arcs meeting only at their nonseparating points.

The point $x \in X$ is an end point if it is a nonseparating point of each arc in $X$ containing $x$. The point $x \in X$ is an arc-cut point if $X-\{x\}$ is not arcwise connected. Note that in locally connected metric continua separating points and arc-cut points are identical. 
THEOREM 1. The continuum $X$ is cyclicly connected if and only if $X$ is arcwise connected and no point in $X$ is an arc-cut point or an end point.

Proof. Clearly the conditions are necessary. To see that they are sufficient define, for each $x \in X, C(x)$ to be the union of all simple closed curves in $X$ containing $x$. The proof is divided into three parts: (i) for each $x \in X, C(x) \neq \varnothing$; (ii) $\{C(x) \mid x \in X\}$ is a decomposition of $X$; and (iii) for each $x \in X, C(x)=X$.

(i) Fix a point $p \in X$. Since $p$ is not an end point there exists an $\operatorname{arc} A$ from $a$ to $b$ in $X$ with $p \in A-\{a, b\}$. Since $p$ does not arc-cut $X$, there exists an arc $B \subseteq X-\{p\}$ from $a$ to $b$. On the arc $A$, in the natural order < from $a$ to $b$, let $a^{\prime}=\operatorname{lub}\{x \in B \mid x \leqslant p\}$ and $b^{\prime}=\operatorname{glb}\{x \in B \mid p \leqslant x\}$. If $A^{\prime}$ (respectively $B^{\prime}$ ) is the arc in $A$ (respectively $B$ ) from $a^{\prime}$ to $b^{\prime}$, then $A^{\prime} \cup B^{\prime}$ is a simple closed curve in $X$ containing $p$. Thus, $C(p) \neq \varnothing$.

(ii) (This part of the argument is similar to that originally employed by Whyburn [12].) It suffices to prove: If $J \subseteq X$ is a simple closed curve and $J \cap C(p) \neq \varnothing$, then $J \subseteq C(p)$. Fix a point $x \in J \cap C(p)$ and a simple closed curve $K$ containing $p$ and $x$. If $J \cap K$ is nondegenerate it is easily seen that for any point $z \in J$ there exists in $J \cup K$ a simple closed curve containing $p$ and $z$. If $J \cap K=\{x\}$ then let $A \subseteq X-\{x\}$ be an arc irreducible from $J$ to $K$. Again, it is easy to see that for each $z \in J$ there exists in $J \cup K \cup A$ a simple closed curve containing $p$ and $z$. Thus $J \subseteq C(p)$, as required.

(iii) Suppose for some $p \in X, C(p) \neq X$. Choose $q \in X-C(p)$; then $C(p) \cap$ $C(q)=\varnothing$. Let $A \subseteq X$ be an arc with nonseparating points $a$ and $b$ satisfying $p$, $q \in A^{\prime} \equiv A-\{a, b\}$. (Such an arc is contained in the union of a simple closed curve containing $p$, a simple closed curve containing $q$, and an arc from $p$ to $q$.) For each $x \in A^{\prime}$, let $B_{x} \subseteq X-\{x\}$ be an arc from $a$ to $b$. As constructed in (i), let $J_{x} \subseteq A \cup B_{x}$ be a simple closed curve such that $J_{x} \cap A$ is an arc with $x$ in its relative interior. It follows that for each $x \in A^{\prime}, C(x) \cap A^{\prime}$ is open in $A^{\prime}$. Since $p$, $q \in A^{\prime},\left\{C(x) \cap A^{\prime} \mid x \in A^{\prime}\right\}$ is a nondegenerate covering of the connected $A^{\prime}$ by pairwise disjoint sets open in $A^{\prime}$, which is impossible.

The Baire Property and the Effros Theorem. In this section $X, d$ denotes a separable metric space. For each $x \in X$ and $\varepsilon>0, B(\varepsilon, x)$ is the $\varepsilon$-ball in $X$ about $x$ and $A^{0}[\bar{A}, \partial A]$ is the interior [closure, boundary] of the subset $A \subseteq X$.

The quasi-interior $A^{*}$ of $A \subseteq X[3]$ is the union of all open subsets $G \subseteq X$ with $G-A$ meager ( $\equiv$ of the first category in $X$ ). If there exists an open subset $G \subseteq X$ with $G-A$ and $A-G$ meager then $A$ is said to have the Baire Property $([9$, p. 87] or [7, p. 210]). We remark, for future use, that arccomponents of closed subsets of $X$ have the Baire Property ([10, Corollary 1.7] and [9, Corollary 1, p. 482]). Note that the Baire Property in the restricted sense, as defined by Kuratowski, is stronger than the Baire Property itself.

Lemma 1. Let $A \subseteq X$. Then (i) $A^{*}-A$ is meager, (ii) $A$ has the Baire Property if and only if $A-A^{*}$ is meager, (iii) if $A$ has the Baire Property and is nonmeager, then $A^{*} \neq \varnothing$. 
Proof. (i) Since $X$ is separable metric, $A^{*}$ can be written as the countable union of open sets $G \subseteq X$ with $G-A$ meager. Hence $A^{*}-A$ is the countable union of meager sets. (ii) Since $A^{*}$ is open, if $A^{*}-A$ is meager (i) implies $A$ has the Baire Property. Conversely, assume $A$ has the Baire Property and let $G \subseteq X$ be an open set with $G-A$ and $A-G$ meager. Then $A-A^{*}$ is a subset of the meager $A-G$ and hence is itself meager. (iii) follows immediately from (ii).

Lemma 2. Suppose $A \subseteq X$ and $h: X \rightarrow X$ is an automorphism (三 self-homeomorphism). If $h\left(A^{*}\right) \cap A^{*} \neq \varnothing$, then $h(A) \cap A \neq \varnothing$.

Proof. Assume $h(A) \cap A=\varnothing$. Observe that $\left(h\left(A^{*}\right) \cap A^{*}\right)-A$ and $\left(h\left(A^{*}\right) \cap\right.$ $\left.A^{*}\right)-h(A)$ are meager since the former is a subset of $A^{*}-A$ and the latter a subset of $h\left(A^{*}\right)-h(A)=h\left(A^{*}-A\right)$. But this demands that their union $\left[\left(h\left(A^{*}\right)\right.\right.$ $\left.\left.\cap A^{*}\right)-h(A)\right] \cup\left[\left(h\left(A^{*}\right) \cap A^{*}\right)-A\right]=\left(h\left(A^{*}\right) \cap A^{*}\right)-(h(A) \cap A)=h\left(A^{*}\right) \cap$ $A^{*}$ is meager. This contradicts the Baire Category Theorem since $h\left(A^{*}\right) \cap A^{*}$ is nonempty and open in $X$.

The following theorem due to E. G. Effros [3] has proven to be very useful in the study of homogeneous continua.

THEOREM 2 [EFFros]. Let $X, d$ be a homogeneous continuum and $\varepsilon>0$. Then there exists $\delta>0$ such that for all $x, y \in X$ with $d(x, y)<\delta$ there exists a (pointed) automorphism $h:(X, x) \rightarrow(X, y)$ satisfying $z \in h(B(\varepsilon, z))$ for each $z \in X$.

We shall refer to $\delta$ and $h$ as an Effros number for $\varepsilon$ and an $\varepsilon$-automorphism, respectively.

LemMa 3. Let $X, d$ be a homogeneous continuum. Suppose $U \subseteq X$ is open, $K$ is an arccomponent of $X-U, V \subseteq \bar{V} \subseteq U$, and $L$ is the arc-component of $X-V$ containing $K$. If $K^{*} \neq \varnothing$, then $\bar{K} \subseteq L^{0}$.

Proof. Fix a point $p \in \bar{K}$. To show $p \in L^{0}$ we will construct an arcwise connected neighborhood $N$ of $p$ contained in $X-V$.

Fix $x \in K^{*}$ and $\varepsilon>0$ satisfying $B(\varepsilon, x) \subseteq K^{*}$ and $\varepsilon<d(\bar{V}, X-U)$. Choose an Effros number $\delta$ for $\varepsilon$ and a point $z \in B(\delta / 2, p) \cap K$. For each $y \in B(\delta / 2, p)$ let $h_{y}:(X, z) \rightarrow(X, y)$ be an $\varepsilon$-automorphism. Finally, define

$$
N=\bigcup\left\{h_{y}(K) \cup K \mid y \in B(\delta / 2, p)\right\}
$$

Let $y \in B(\delta / 2, p)$. Since $y=h_{y}(z) \in h_{y}(K) \subseteq N$ it follows that $B(\delta / 2, p) \subseteq N$; i.e., $N$ is a neighborhood of $p$. Since $x \in h_{y}(B(\varepsilon, x)) \subseteq h_{y}\left(K^{*}\right)$ we have that $h_{y}\left(K^{*}\right) \cap K^{*} \neq \varnothing$ and hence, by Lemma $2, h_{y}(K) \cap K \neq \varnothing$. Thus, $N$ is arcwise connected. The second requirement on $\varepsilon$ implies that $h_{y}(K) \subseteq X-V$ which yields $N \subseteq X-V$. Since $K \subseteq N$ we have $p \in N^{0} \subseteq N \subseteq L$ which completes the proof of the lemma.

On arcwise connected homogeneous continua. Let $X, d$ denote a fixed, but arbitrary, arcwise connected homogeneous metric continuum. The main result in this section is that $X$ has no arc-cut points and hence is cyclicly connected. We begin by defining a set-valued function similar to F. B. Jones' function $K$ [5]. 
For each $x \in X$, let $R(x)=\cap\{\bar{N} \mid N \in \mathcal{N}(x)\}$ where $\Re(x)$ is the set of all arcwise connected neighborhoods of $x$. Clearly, each $R(x)$ is closed and nonempty.

LEMMA 4. For each $x \in X$,

$$
R(x)=\bigcap\{N \mid N \in \Re(x)\}=\bigcap\left\{N^{0} \mid N \in \mathscr{U}(x)\right\} .
$$

Proof. For notational convenience let $Q(x)=\cap\{N \mid N \in \mathfrak{T}(x)\}$ and $P(x)=$ $\cap\left\{N^{0} \mid N \in \mathcal{K}(x)\right\}$. Since $P(x) \subseteq Q(x) \subseteq R(x)$ it suffices to verify (i) $Q(x) \subseteq$ $P(x)$ and (ii) $X-Q(x) \subseteq X-R(x)$.

To prove (i) we show: If $y \in Q(x)$ and $N \in \mathcal{N}(x)$ then $y \in N^{0}$. Suppose not; then $y \in N-N^{0}$. Let $\varepsilon>0$ be such that $B(\varepsilon, x) \subseteq N$. Choose an Effros number $\delta$ for $\varepsilon$, a point $z \in B(\delta, y) \cap(X-N)$, and an $\varepsilon$-automorphism $h:(X, z) \rightarrow(X, y)$. Then $x \in h(B(\varepsilon, x)) \subseteq h(N)$; i.e., $h(N) \in \Re(x)$. However, $y=h(z) \in h(X-N)$ $=X-h(N)$ which contradicts the assumption that $y \in Q(x)$.

To prove (ii) fix a point $y \in X-Q(x)$. We will use Lemma 3 to construct an arcwise connected neighborhood of $x$ whose closure misses $y$. Assume, without loss of generality, that $d(x, y)>1$. For each $n=1,2, \ldots$ let $A_{n}$ denote the arccomponent of $X-B(1 / n, y)$ containing $x$. Since $y \in X-Q(x)$ there exists $N \in \mathfrak{K}(x)$ with $N \subseteq X-\{y\}$. Thus, $A=\cup\left\{A_{n} \mid n=1,2, \ldots\right\}$, the arccomponent of $X-$ $\{y\}$ containing $x$, has nonempty interior. In particular, $A$ is nonmeager and hence some $A_{m}$ is nonmeager. Since $A_{m}$ also has the Baire Property, we infer from Lemma 1 that $A_{m}^{*} \neq \varnothing$. By Lemma 3, with $U=B(1 / m, y), K=A_{m}, V=B(1 /(m+1), y)$, and $L=A_{m+1}$, we see that $A_{m+1}$ is an arcwise connected neighborhood of $x$ whose closures misses $y$. That is, $y \in X-R(x)$, concluding the proof of (ii).

Lemma 5. If $y \in R(x)$, then $R(y)=R(x)$.

Proof. It follows easily from Lemma 4 that if $y \in R(x)$ then $R(y) \subseteq R(x)$. For the reverse inclusion we find a point $x_{0} \in X$ such that $y \in R\left(x_{0}\right)$ implies $R\left(x_{0}\right) \subseteq$ $R(y)$. By homogeneity $x_{0}$ can be any point in $X$.

Partially order, with respect to $\subseteq,\{R(x) \mid x \in X\}$ and let $\mathcal{R}$ be a maximal chain. Since each $R(x)$ is nonempty and closed, there exists a point $x_{0} \in \cap \Re$. By the maximality of $R$ it follows that $R\left(x_{0}\right)$ is the minimal element of $R$. Hence if $y \in R\left(x_{0}\right), R\left(x_{0}\right) \subseteq R(y)$.

LeMma 6. For each $x \in X, R(x) \neq X$.

Proof. Assume, to the contrary, that $R(x)=X$. Then from Lemma 1 we infer that each proper arcwise connected subset of $X$ has empty interior, or equivalently, dense complement. Fix a point $p \in X$ and let $\left\{U_{n} \mid n=1,2, \ldots\right\}$ be a countable base for $X-\{p\}$ with each $U_{n} \neq \varnothing$. For each $n$ denote by $A_{n}$ the arccomponent of $X-U_{n}$ containing $p$. Then each $A_{n}$ has the Baire Property and hence, by Lemma $1, A_{n}-A_{n}^{*}$ is meager. Observe that for some $n, A_{n} \cap A_{n}^{*} \neq \varnothing$; for if not, $A_{n}-A_{n}^{*}=A_{n}$ is meager for each $n$, as is $X=\cup\left\{A_{n} \mid n=1,2, \ldots\right\}$ contradicting the Baire Category Theorem. Assume, without loss of generality, that $A_{1} \cap A_{1}^{*} \neq$ $\varnothing$ (and hence, $A_{1}^{*} \neq \varnothing$ ). Choose $m$ such that $U_{m} \subseteq \bar{U}_{m} \subseteq U_{1}$. Then, by Lemma 3, $p \in \bar{A}_{1} \subseteq A_{m}^{0} \neq \varnothing$, a contradiction. Thus $R(x) \neq X$, as required. 
Lemma 7. For each $x \in X, R(x)$ is connected.

Proof. Suppose $R(x)=A \cup B$ where $A$ and $B$ are nonempty disjoint closed sets with $x \in A$. Fix a point $y \in B$. A contradiction will be obtained as follows. We first construct a neighborhood $M \subseteq \bar{M} \subseteq X-\{x\}$ of $y$ with only finitely many, say $n$, arccomponents. If $n \geqslant 2$, we use the Effros Theorem to define a neighborhood $N \subseteq \bar{N} \subseteq X-\{x\}$ of $y$ with at most $n-1$ arccomponents. Induction then yields an arcwise connected neighborhood of $y$ missing $x$. Thus $x \in R(y)$, or equivalently $y \in R(x)$.

To construct the neighborhood $M$ let $V$ be an open set satisfying $B \subseteq V \subseteq \bar{V} \subseteq$ $X-A \subseteq X-\{x\}$. For each $z \in \partial V$ let $N(z)$ be an arcwise connected neighborhood of $z$ whose closure misses $x$. Let $D \subseteq \partial V$ be a finite set such that $\partial V \subseteq$ $\cup\{N(z) \mid z \in D\}$ and define $M=\cup\{N(z) \mid z \in D\} \cup V$. Clearly $M$ is a neighborhood of $y$ whose closure misses $x$. Moreover, since each arccomponent of $M$ meets $\partial V$ and each $N(z)$ is arcwise connected, $M$ has only finitely many arccomponents, $A_{1}, A_{2}, \ldots, A_{n}$.

Now suppose $n \geqslant 2$. Assume, without loss of generality, that $y \in A_{1}$. If $y \in A_{1}^{0}$ then $A_{1}$ is an arcwise connected neighborhood of $y$ missing $x$ and we are done. If $y \notin A_{1}^{0}$ then $y \in \overline{M-A_{1}} \subseteq \cup\left\{\bar{A}_{i} \mid i=2,3, \ldots, n\right\}$. Assume, without loss of generality, that $y \in \overline{A_{2}}$. For each $i$ fix a point $z_{i} \in D$ such that $N\left(z_{i}\right) \subseteq A_{i}$. Choose $\varepsilon>0$ such that $B\left(\varepsilon, z_{i}\right) \subseteq N\left(z_{i}\right)$ for each $i$, and the closure of $B(\varepsilon, \bar{M})$ misses $x$. Fix a point $z \in B(\delta, y) \cap A_{2}$ where $\delta$ is an Effros number for $\varepsilon$ and $h:(X, z) \rightarrow$ $(X, y)$ an $\varepsilon$-automorphism. Then $x \notin h(\bar{M})$ and hence $N=M \cup h(M)=$ $\cup\left\{A_{i} \cup h\left(A_{i}\right) \mid i=1,2, \ldots, n\right\}$ is a neighborhood of $y$ whose closure misses $x$. Since $h\left(z_{i}\right) \in B\left(\varepsilon, z_{i}\right) \cap h\left(A_{i}\right) \subseteq A_{i} \cap h\left(A_{i}\right)$, each $A_{i} \cup h\left(A_{i}\right)$ is arcwise connected. Moreover, since $z=h(y) \in h\left(A_{1}\right) \cap A_{2},\left(A_{1} \cup h\left(A_{1}\right)\right) \cup\left(A_{2} \cup h\left(A_{2}\right)\right)$ is arcwise connected. Consequently, $N$ has at most $n-1$ arccomponents. This completes the proof of the lemma as outlined above.

The next lemma is a special case of a more general theorem due to J. T. Rogers, Jr. [11]. We include a proof for the sake of completeness.

LeMma 8. $\{R(x) \mid x \in X\}$ is a continuous monotone decomposition of $X$.

Proof. In view of Lemmas 5 and 7 it suffices to show the function $x \mapsto R(x)$ is continuous from $X$ into the hyperspace of subcontinua of $X$ with the Hausdorff metric $\rho$. Suppose $\left\{x_{n}\right\}$ is a sequence in $X$ converging to $x$ and $\varepsilon>0$. Choose an Effros number $\delta$ for $\varepsilon$ and $N>0$ such that for all $n \geqslant N, d\left(x_{n}, x\right)<\delta$. For each $n \geqslant N$ let $h_{n}:(X, x) \rightarrow\left(X, x_{n}\right)$ be an $\varepsilon$-automorphism. Then since $h_{n}(R(x))=$ $R\left(h_{n}(x)\right)=R\left(x_{n}\right)$ and $h_{n}$ moves no point more than $\varepsilon$ it follows that $\rho\left(R\left(x_{n}\right), R(x)\right)$ $<\varepsilon$.

Lemma 9. For each $x \in X, X-R(x)$ is connected.

Proof. From Lemma 8 and the fact that each automorphism on $X$ induces a permutation on $\{R(x) \mid x \in X\}$ we have: the quotient space $X / R$ is a homogeneous metric continuum and the quotient map $\pi: X \rightarrow X / R$ is monotone. Thus, $X-$ $R(x)=\pi^{-1}(X / R-\{R(x)\})$ is connected. 
TheOREM 3. Arcwise connected homogeneous metric continua contain no arc-cut points. Consequently, such continua are cyclicly connected.

Proof. Let $X$ be as before and fix $x \in X$. Observe that some point in $R(x)$ is arcwise-accessible from $X-R(x)$ and hence, by homogeneity, every point in $R(x)$ has this property. In particular, for each $y \in R(x)-\{x\}$ there exists a nondegenerate arc whose intersection with $R(x)$ is $\{y\}$. To show $X-\{x\}$ is arcwise connected then, it suffices to prove: For each pair of points $a, b \in X-R(x)$ there exists an arc $A \subseteq X-\{x\}$ from $a$ to $b$. But this follows from an elementary chaining argument since $X-R(x)$ is connected and can be covered by arcwise connected neighborhoods missing $x$.

The last statement follows immediately from Theorem 1.

Corollary 1. For each $x \in X, R(x)=\{x\}$.

We conclude with some related questions. Wilson's original question is still of interest.

(1) [Wilson] Does there exist a uniquely arcwise connected homogeneous Hausdorff continuum?

More generally one might ask

(2) Which of the theorems of this paper are true in Hausdorff continua?

(3) Suppose $X$ is an arcwise connected homogeneous metric continuum which is itself not a simple closed curve. Does $X$ contain simple closed curves of arbitrarily small diameter?

In conversation with the authors G. R. Gordh, Jr. raised the following question:

(4) [Gordh] Suppose $X$ is an arcwise connected homogeneous metric continuum and some pair of its points separates $X$. Is $X$ necessarily a simple closed curve?

Finally, we include a question which appears in [8].

(5) [K. Kuperberg] Are arcwise connected homogeneous continua locally connected?

\section{BIBLIOGRAPHY}

1. W. L. Ayres, Concerning continuous curves in metric space, Amer. J. Math. 51 (1929), 577-594.

2. __ A new proof of the cyclic connectivity theorem, Bull. Amer. Math. Soc. 48 (1942), 627-630.

3. E. G. Effros, Transformation groups and $C^{*}$-algebras, Ann. of Math. (2) 81 (1965), 38-55.

4. C. L. Hagopian, Concerning arcwise connectedness and the existence of simple closed curves in plane continua, Trans. Amer. Math. Soc. 147 (1970), 389-402. [See also Trans. Amer. Math. Soc. 157 (1971), 507-509.]

5. F. B. Jones, Concerning non-aposyndetic continua, Amer. J. Math 70 (1948), 403-413.

6. __ The cyclic connectivity of plane continua, Pacific J. Math. 2 (1961), 1013-1016.

7. J. L. Kelley, General topology, Van Nostrand, New York, 1952.

8. K. Kuperberg, A locally connected micro-homogeneous non-homogeneous continuum, Bull. Acad. Polon. Sci. (to appear).

9. K. Kuratowski, Topology. I, PWN, Warsaw; Academic Press, New York, 1966.

10. L. K. Mohler, The fixed point property for homeomorphisms of 1-arcwise connected continua, Proc. Amer. Math. Soc. 52 (1975), 451-456. 
11. J. T. Rogers, Decompositions of homogeneous continua (preprint).

12. G. T. Whyburn, Cyclicly connected continuous curves, Proc. Nat. Acad. Sci. U.S.A. 13 (1927), 31-38.

13. On the cyclic connectivity theorem, Bull. Amer. Math. Soc. 37 (1931), 429-433.

14. R. L. Wilson, Intrinsic topologies on partially ordered sets and results on compact semigroups, Ph.D. dissertation, Univ. Tennessee, 1978.

Department of Mathematics, University of Delaware, Newark, Delaware 19711

Department of Mathematics, Salem College, Winston-Salem, North Carolina 27108 